\title{
Extraordinary Exciton Conductance Induced by Strong Coupling
}

\author{
Johannes Feist ${ }^{1, *}$ and Francisco J. Garcia-Vidal ${ }^{1,2, \dagger}$ \\ ${ }^{1}$ Departamento de Física Teórica de la Materia Condensada and Condensed Matter Physics Center (IFIMAC), \\ Universidad Autónoma de Madrid, E-28049 Madrid, Spain \\ ${ }^{2}$ Donostia International Physics Center (DIPC), E-20018 Donostia/San Sebastian, Spain
}

(Received 8 September 2014; published 12 May 2015)

\begin{abstract}
We demonstrate that exciton conductance in organic materials can be enhanced by several orders of magnitude when the molecules are strongly coupled to an electromagnetic mode. Using a 1D model system, we show how the formation of a collective polaritonic mode allows excitons to bypass the disordered array of molecules and jump directly from one end of the structure to the other. This finding could have important implications in the fields of exciton transistors, heat transport, photosynthesis, and biological systems in which exciton transport plays a key role.
\end{abstract}

The transport of excitons (bound electron-hole pairs) is a fundamental process that plays a crucial rule both in natural phenomena such as photosynthesis, where energy has to be transported to a reaction center [1-3], and in artificial devices such as excitonic transistors [4,5] or organic solar cells, whose power conversion efficiency can be improved significantly when the exciton diffusion length is increased [6]. Similarly, understanding and manipulating the role of excitons in heat transport has become an active field of research, with possible applications ranging from thermoelectric effects to heat-voltage converters, to nanoscale refrigerators, and even thermal logic gates (cf. [7] and references therein). The exciton transport efficiency depends on a wide range of factors with such surprising features as the occurrence of noise-assisted transport [8-10]. Pioneering works have even suggested that coherent transport can play an important role in biological systems $[2,3,11]$. However, most systems composed of organic molecules are disordered and possess relatively large dissipation and dephasing rates, such that exciton transport typically becomes diffusive over long distances [12].

An intriguing possibility to modify exciton properties is by strong coupling to an electromagnetic (EM) mode, forming so-called polaritons (hybrid light-matter states). This is achieved when the Rabi frequency, i.e., the energy exchange rate between exciton and EM modes, becomes faster than the decay and/or decoherence rates of either constituent. Polaritons combine the properties of their constituents, in particular, mutual interactions and low effective masses, enabling new applications such as polariton condensation in semiconductors [13] and organic materials [14], the modification of molecular chemistry [15] and work functions [16], or the transfer of excitation between different molecular species [17]. Because of the large dipole moments and high densities, organic materials support large Rabi splittings [18-20], and can also be strongly coupled to surface plasmon polaritons [19,21-24]. The dispersion relation can then be tuned to achieve a further reduction of the effective mass [25].
Very recently, an increase of the electrical conductance of an organic material was shown under strong coupling of the excitons to a cavity mode [26]. Inspired by this result, we demonstrate in this Letter that through strong coupling to an electromagnetic mode, i.e., the creation of polaritonic states, the exciton transport efficiency can be improved by many orders of magnitude. The strong coupling allows the excitons to bypass the disordered organic system, preventing localization and leading to dramatically improved energy transport properties. We note that while we focus on organic molecules in the following, the results can readily be generalized to other systems such as quantum dots and Rydberg atoms, or even chains of trapped ions, which offer a high degree of controllability $[27,28]$.

We focus on a model system that captures the essential physics: A 1D chain of two-level emitters inside a cavity (see Fig. 1). The emitter dipole transition is coupled to the single cavity mode, and, additionally, induces Coulombic dipole-dipole interaction between the emitters. The effect of internal (e.g., rovibrational or phononic) and external environment modes is taken into account through effective dephasing and nonradiative decay rates modeled using a master equation of Lindblad form. The system Hamiltonian

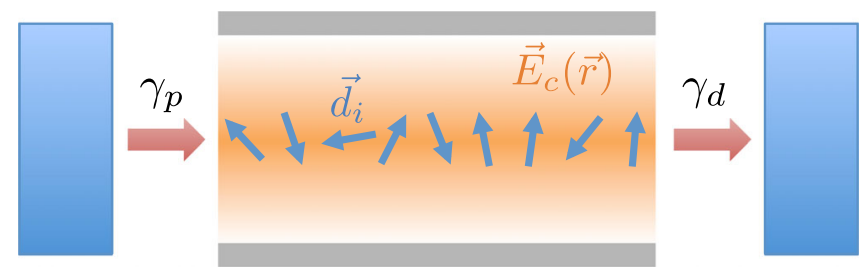

FIG. 1 (color online). Sketch of the model system: A 1D chain of (possibly disordered) quantum emitters with dipole moments $\vec{d}_{i}$ inside a cavity with cavity mode $\vec{E}_{c}(\vec{r})$. Excitons are pumped into the system from the left reservoir with rate $\gamma_{p}$. The exciton current is measured by the excitons reaching the sink reservoir on the right, coupled through incoherent decay of the last emitter with rate $\gamma_{d}$. 
$H$ in the rotating wave approximation (setting $\hbar=1$ here and in the following) is then

$$
\begin{aligned}
H= & \omega_{c} a^{\dagger} a+\sum_{i} \omega_{m} \sigma_{i}^{+} \sigma_{i}^{-}+\sum_{i} g_{i}\left(a^{\dagger} \sigma_{i}^{-}+a \sigma_{i}^{+}\right) \\
& +\sum_{i, j} V_{i j}^{d d}\left(\sigma_{i}^{+} \sigma_{j}^{-}+\sigma_{j}^{+} \sigma_{i}^{-}\right),
\end{aligned}
$$

where $a$ is the bosonic annihilation operator of the cavity mode with energy $\omega_{c}$ and electric field $\vec{E}_{c}(\vec{r})$. The molecular excitons of energy $\omega_{m}$ are created and destroyed by the fermionic operators $\sigma_{i}^{+}$and $\sigma_{i}^{-}$. Molecule $i$ is characterized by its position $\vec{r}_{i}$ and dipole moment $\vec{d}_{i}$, giving the cavity-molecule interaction $g_{i}=-\vec{d}_{i} \cdot \vec{E}_{c}\left(\vec{r}_{i}\right)$. The dipole-dipole interaction (in the quasistatic limit) is

$$
V_{i j}^{d d}=\frac{\vec{d}_{i} \cdot \vec{d}_{j}-3\left(\vec{d}_{i} \cdot \hat{R}_{i j}\right)\left(\vec{d}_{j} \cdot \hat{R}_{i j}\right)}{4 \pi \epsilon_{0} R_{i j}^{3}},
$$

with $R_{i j}=\left|\vec{r}_{i}-\vec{r}_{j}\right|$ and $\hat{R}_{i j}=\left(\vec{r}_{i}-\vec{r}_{j}\right) / R_{i j}$.

The system dynamics is described by a Lindblad master equation for the density matrix

$$
\dot{\rho}=-i[H, \rho]+\sum_{\alpha=i, c} \mathcal{L}_{\alpha}[\rho],
$$

where $\alpha$ runs over all molecules as well as the cavity mode. The superoperators $\mathcal{L}_{\alpha}$ describe decay and dephasing:

$$
\begin{gathered}
\mathcal{L}_{i}=\gamma_{d} L_{\sigma_{i}^{-}}+\gamma_{\phi} L_{\sigma_{i}^{+} \sigma_{i}^{-}}, \\
\mathcal{L}_{c}=\kappa L_{a},
\end{gathered}
$$

where $L_{c}[\rho]=c \rho c^{\dagger}-\frac{1}{2}\left\{c^{\dagger} c, \rho\right\}$ is the standard form for Lindblad superoperators. The total molecule decay rate $\gamma_{d}$ is given by $\gamma_{d}=\gamma_{r}+\gamma_{n r}$, with $\gamma_{r}$ and $\gamma_{n r}$ the radiative and nonradiative decay rates, while $\gamma_{\phi}$ is the dephasing rate. The decay rate $\kappa$ of the cavity photons is dominated by leakage through the mirrors. For later reference, we also define the total molecular decoherence rate $\gamma=\gamma_{d}+\gamma_{\phi}$. We note that while molecular decay and dephasing as included here models exciton-phonon interactions, it cannot represent exciton self-trapping, which can be approximated by a nonlinear term in the energy functional [29,30]. Selftrapping can lead to a further reduction of the exciton propagation length in the weakly coupled limit, but we have checked that it does not significantly affect the strongly coupled limit. We thus neglect it in the following.

As mentioned above, we only include one cavity mode and describe the molecules by a linear 1D chain along the longitudinal cavity direction ( $x$ axis), with positions $\vec{r}_{i}=x_{i} \hat{x}$, such that the cavity electric field is identical for all molecules. It is polarized along the (out-of-plane) $z$ axis, leading to $\vec{E}_{c}\left(\vec{r}_{i}\right)=E_{c} \hat{z}$. The total coupling between molecules and the cavity mode can be characterized by the collective Rabi frequency $\Omega_{R}=2 \sqrt{\sum_{i} g_{i}^{2}}$. For zero detuning $\omega_{m}=\omega_{c}$, strong coupling is entered for $\Omega_{R}>|\gamma-\kappa| / 2$ and leads to the formation of upper and lower polaritons at energies $\omega_{m} \pm(1 / 2) \sqrt{\Omega_{R}^{2}-|\gamma-\kappa|^{2} / 4}$. The Rabi splitting (energy difference between upper and lower polariton) can approach $1 \mathrm{eV}$ in experiments [19,20], and can be tuned by changing either the molecule density or the mode electric field strength.

In the following, we consider two types of molecular configurations: A perfectly ordered distribution, with molecule positions on a regular grid and dipole moments perfectly aligned to the electric field (i.e., along the $z$ axis), and a random distribution, where Gaussian noise is added to the regular positions and the dipole moments are oriented randomly. Note that for the present case of a 1D linear chain, randomness is expected to suppress conductance much more efficiently than in higher dimensions.

We next introduce a prescription for calculating an exciton conductance $\sigma_{e}$, a steady-state quantity to characterize the exciton transport efficiency similar to the electrical conductance for charge transport. We assume that excitation is continuously pumped into the system on the left side and measure the energy leaving the system through the right side (cf. Fig. 1). The pumping is represented by an additional incoherent driving term $\mathcal{L}_{p}=\gamma_{p} L_{\sigma_{1}^{+}}$.

The energy current is obtained from the rate of change of energy [31]

$$
\dot{E}=\frac{d}{d t}\langle H\rangle=\operatorname{Tr}(H \dot{\rho})=\sum_{\alpha} \operatorname{Tr}\left(H \mathcal{L}_{\alpha}[\rho]\right) .
$$

This is evaluated in the steady state, $\rho=\rho_{s s}$, for which the total rate of change in energy is zero $\left(\dot{\rho}_{s s}=0 \rightarrow \dot{E}=0\right)$. However, each of the Lindblad superoperators $\mathcal{L}_{\alpha}$ can be associated with a specific physical process. We thus identify the energy current between the two reservoirs with the loss of energy from the last molecule:

$$
J=\gamma_{d} \operatorname{Tr}\left(H L_{\sigma_{N}^{-}}\left[\rho_{s s}\right]\right) .
$$

We then define the exciton conductance as the current per driving power, i.e., $\sigma_{e}=J / \gamma_{p}$ (which has units of energy). Note that contrary to [31], the energy entering the system through pumping does not necessarily leave through the sink at the end. It can also be lost through the radiative and nonradiative decay of the molecules, as well as decay of the cavity mode.

We numerically obtain the steady state of the system using the open-source QUTIP package [32]. To do so, we restrict the total superoperator in Eq. (3) to the zero- and single-excitation subspaces. This truncation of the Hilbert space is an excellent approximation in the presently relevant linear response regime of weak pumping, i.e., for $\gamma_{p}$ smaller than the system decay rates.

We choose the quantum emitter parameters to approximately correspond to TDBC $J$ aggregates at room temperature [33-35]: $\omega_{m}=2.11 \mathrm{eV}, \gamma_{r}^{-1}=500 \mathrm{ps}, \gamma_{n r}^{-1}=600 \mathrm{fs}$, and $\gamma_{\phi}^{-1}=25$ fs. The cavity lifetime $\kappa^{-1}=50 \mathrm{fs}$ is typical for experiments using cavities made of thin metal mirrors [26]. The molecule parameters also determine the dipole moment through $\gamma_{r}=\omega_{m}^{3} d^{2} /\left(3 \pi \epsilon_{0} \hbar c^{3}\right)$, giving $d \approx 36 \mathrm{D}$. 
The average intermolecular spacing is taken as $\delta x=3 \mathrm{~nm}$. Note that while the coupling to the cavity mode is taken into account explicitly, the radiative decay rate into all other electromagnetic modes can also be modified by the presence of a cavity. Since $\gamma_{r}$ is much smaller than the other rates, this modification can safely be neglected here. While we only show results for the parameters given above, we checked that the main conclusions drawn in the following apply for a wide range of parameters and do not depend on the specific values chosen here.

Figure 2 shows the exciton conductance $\sigma_{e}$ at zero cavitymolecule detuning $\omega_{c}=\omega_{m}$, as a function of the collective Rabi frequency $\Omega_{R}$. Here, we keep the number of molecules fixed and change the electric field strength, going through the transition from weak to strong coupling. This can be achieved in an experiment by, e.g., putting the molecules at different positions inside the cavity [36]. We compare regular and random molecule arrangements for chains of 40 and 60 molecules. For the regular distribution, the strong dipole-dipole interaction leads to an additional small energy shift $\Delta$ of the molecular bright state coupling to the cavity; zero detuning thus corresponds to $\omega_{c}=\omega_{m}+\Delta$.

For all of the cases shown in Fig. 2, the conductance is approximately constant in the weak coupling limit $\Omega_{R} \ll \gamma, \kappa$, where the cavity mode does not play a role. Unsurprisingly, the conductance in this limit strongly depends on the molecular configuration-it is almost completely suppressed for the random case, for which 1D systems always show Anderson localization. The conductance in the random case is calculated as the logarithmic mean of 100 random configurations as appropriate for localized systems, i.e., $\sigma_{e}=\exp \left\langle\log \sigma_{e}^{i}\right\rangle$ [37]. Note that even in the regular case, transport is quite inefficient due to the relatively large decay and dephasing rates of the molecular excitons, leading to diffusive transport $[10,12]$. Strikingly, when the coupling to the cavity mode is

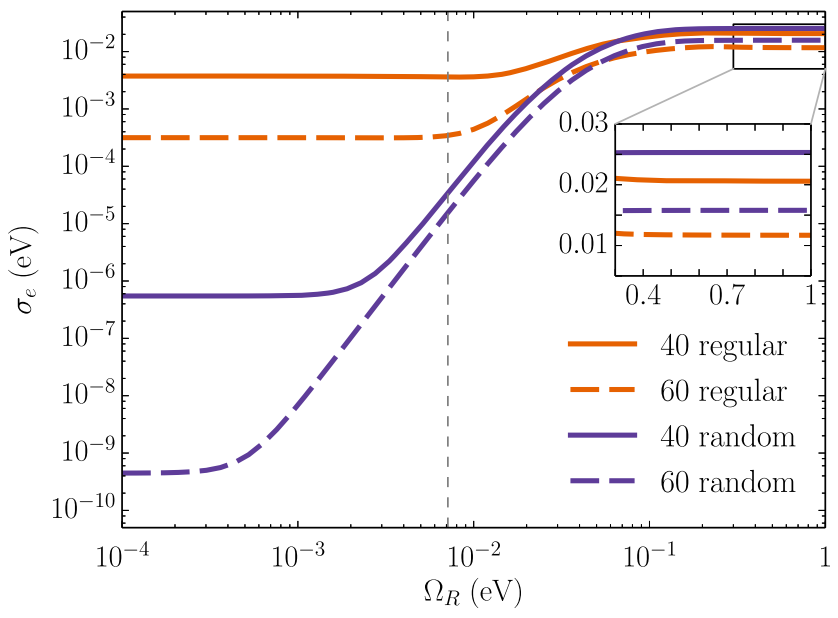

FIG. 2 (color online). Exciton conductance at zero detuning as a function of the Rabi frequency for four different molecular configurations. The thin gray dashed line indicates the onset of strong coupling at $\Omega_{R}=|\gamma-\kappa| / 2$. The number in the label indicates the number of molecules. The inset shows a zoom of the strong-coupling region. increased, an extraordinary increase of the conductance is observed in all cases. Once strong coupling is reached $\left(\Omega_{R} \gg \gamma, \kappa\right)$, the conductance again becomes almost independent of $\Omega_{R}$, indicating that the fully formed polariton channel dominates exciton conductance. In this limit, the conductance also becomes almost independent of the configuration and only depends on the number of molecules, i.e., length of the $1 \mathrm{D}$ chain. While randomness can suppress conduction almost completely in the weak-coupling limit, the polariton modes are barely affected by it. As a consequence of their delocalized nature induced by the collective exciton-cavity coupling, the excitation can efficiently bypass the disordered chain of emitters.

This also provides a possible indication for the mechanism behind the enhanced electrical conduction observed under strong coupling in the experiments by Orgiu et al. [26]. However, the connection between exciton transport through polaritons and electrical conduction is currently unclear, as polaritons are, in principle, neutral quasiparticles.

We also note that while we focus on incoherent driving for simplicity in this work, we have found that under coherent driving at frequency $\omega$, the same general behavior is observed. The main difference is an additional resonant enhancement when $\omega$ coincides with the eigenfrequencies of the system (see details in the Supplemental Material [38]). An interesting aspect is that even when hopping is completely suppressed, resonant transport occurs not only when driving at the polariton eigenfrequencies, but also at the unmodified molecule frequency - a clear signature that the dark states (which are not coupled to the cavity EM field) are still affected by the existence of strong coupling.

We next focus on the case where the cavity mode is detuned from the molecular excitations by an energy $\delta \omega=\omega_{m}-\omega_{c}$. As shown in Fig. 3, the onset of the extraordinary conductance is then shifted to larger coupling strengths for increasing detuning $|\delta \omega|$. However, the final conductance in the strong-coupling limit is

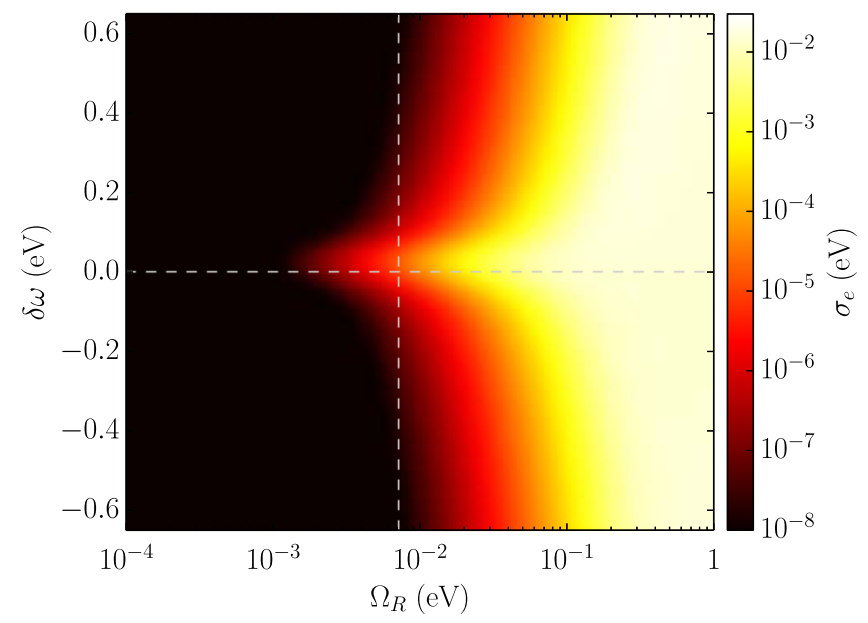

FIG. 3 (color online). Conductance as a function of Rabi splitting and detuning for random configurations of 60 emitters (averaged over 100 random realizations). The thin gray dashed lines indicate $\delta \omega=0$ and the onset of strong coupling at $\Omega_{R}=|\gamma-\kappa| / 2$. 
independent of the detuning. This again indicates that the conduction proceeds through the polariton modes, which are only fully formed when the Rabi frequency $\Omega_{R}$ becomes large enough to not only overcome decoherence processes, but also the detuning. Once this is fulfilled, their character does not strongly depend on the detuning. However, even for relatively large and experimentally relevant Rabi frequencies, e.g., $\Omega_{R}=100 \mathrm{meV}$, a small change of the detuning can strongly suppress or enhance conductance. If the detuning could be modified dynamically in an experiment (e.g., by displacing a cavity mirror with a piezo), this would enable novel applications based on switching of the exciton conductance.

Next, we develop a simplified model to understand the extraordinary increase in exciton conductance under strong coupling. The main idea behind it is that there are two almost independent transport channels: (i) Direct excitonic transport through hopping between the molecules, which dominates in the weak-coupling limit, and (ii) polaritonic transport through the collective modes created by strong coupling to the cavity, which increases rapidly (polynomially) as the coupling is increased and saturates in the strong-coupling limit. To expose the contribution of the second channel unambiguously, we remove the dipoledipole interaction responsible for hopping from the Hamiltonian Eq. (1). We furthermore assume that the molecules are all aligned along the cavity field polarization axis $z$. The interaction with the cavity mode is then identical for all molecules.

Figure 4 shows that the picture of independent channels is indeed valid: For the conductance $\sigma_{e}^{\mathrm{NH}}$ without hopping, the transmission plateau for weak coupling disappears,

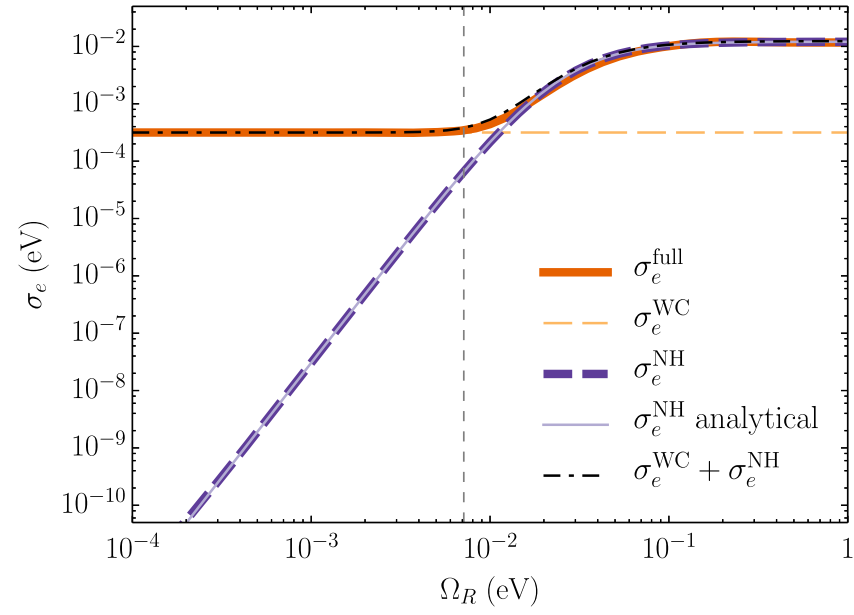

FIG. 4 (color online). Transmission for a regular chain of 60 emitters. The solid orange line gives the results for the full model, with its weak-coupling limit $\sigma_{e}^{\mathrm{WC}}$ indicated by the thin dashed orange line. The result without hopping (dashed purple line), i.e., with dipole-dipole interactions turned off, is perfectly reproduced by the analytical result Eq. (8) (thin light purple line). The dashdotted black line is the sum of the two independent transport channels of direct hopping in the weak-coupling limit and the cavity-mediated contribution without hopping (see text). The vertical gray dashed line again indicates the onset of strong coupling, $\Omega_{R}=|\gamma-\kappa| / 2$. while the transmission in strong coupling is essentially unchanged. The polariton contribution without hopping decreases rapidly with decreasing Rabi frequency. The independence of the two transport channels is further verified by plotting the sum of the exciton conductance in the weak-coupling limit, $\sigma_{e}^{\mathrm{WC}}=\sigma_{e}^{\text {full }}\left(\Omega_{R}=0\right)$, and the cavity-mediated contribution $\sigma_{e}^{\mathrm{NH}}\left(\Omega_{R}\right)$ without hopping. This sum, given by the dash-dotted black line in Fig. 4, agrees excellently with the full result, indicating that the two transport channels are indeed effectively independent.

Since, in this model all molecules but the first (due to the pumping) are indistinguishable, they behave identically. Independent of the number of molecules $N$, there are thus just 12 independent components of the steady state density matrix within the zero- and single-excitation subspace. This density matrix can then be obtained analytically, and inserting the solution into Eq. (7) gives an explicit formula for the conductance. In the linear response limit of weak driving and for zero detuning $\omega_{c}=\omega_{m}$, it is given by

$$
\begin{aligned}
& \sigma_{e}= \\
& \frac{\gamma \gamma_{d}(\gamma+\Gamma) \omega_{m} \Omega_{R}^{4}}{\left(2 \gamma \gamma_{d} \Gamma N+\left(2 \gamma_{\phi}+\gamma_{d} N\right) \Omega_{R}^{2}\right)\left(\kappa \gamma \gamma_{d} \Gamma N+\left(\kappa \gamma_{\phi}+\gamma_{d} \Gamma N\right) \Omega_{R}^{2}\right)},
\end{aligned}
$$

where we have defined the total decoherence rate $\Gamma=\gamma+\kappa$. More details and the full expression for arbitrary detuning are given in the Supplemental Material [38].

As expected, the analytical solution [Eq. (8)] perfectly matches the numerics (cf. Fig. 4). For small Rabi frequency, the conductance through the polariton modes grows with $\Omega_{R}^{4}$, while it saturates to a constant value for $\Omega_{R} \rightarrow \infty$. For large $N$, this constant value is given by $\gamma \omega_{m} /\left(\gamma_{d} N^{2}\right)$ if $\kappa \gg$ $\gamma$ and by $2 \gamma_{\phi} \omega_{m} /\left(\gamma_{d} N^{2}\right)$ if $\gamma_{\phi} \gg \kappa, \gamma_{d}$. Importantly, the decay with system size is algebraic $\left(N^{-2}\right)$, as opposed to the localized $e^{-N}$ behavior expected in the absence of strong coupling. Note that $N$ occurs here because only a single molecule is connected to each of the baths; for a quasi-1D wire with a transverse extension, the length of the system would be the relevant variable. The dependence on the fourth power of $\Omega_{R}$ in the weak-coupling limit is explained by the rate obtained from two quantum jumps, to the cavity mode and back, with coupling $\propto \Omega_{R}$. Interestingly, Eq. (8) shows that the conductance, at least in the simplified model without disorder or direct hopping, is not directly related to the conventional criterion for the onset of strong coupling where the vacuum Rabi splitting becomes real $\left(\Omega_{R}>|\gamma-\kappa| / 2\right)$. Indeed, the difference $\gamma-\kappa$ does not occur in Eq. (8). Instead, the exciton conductance becomes constant when $\Omega_{R}^{2} \gg 2 \gamma \Gamma$ (for large $N$ ). This is related to large values of the cooperativity $C=\Omega_{R}^{2} / \gamma \kappa$, which can occur even if strong coupling is not fully reached.

To conclude, we have demonstrated that the formation of polariton modes, i.e., strong coupling, can dramatically enhance exciton transport. When the coupling is strong enough and the polaritons are fully formed, the excitons 
can almost completely bypass the chain of quantum emitters and "jump" directly from one end to the other, leading to large exciton conductance. This robust effect persists almost independently of the exact parameters of the system, and most notably occurs efficiently even when the underlying excitonic system is strongly disordered and its transport is completely suppressed due to localization. Through a simple model, we have furthermore shown that transport through direct hopping and through the polariton modes constitute two effectively independent channels, which helps to explain why the polariton conductance is almost independent of the disorder in the system. These results demonstrate a possible pathway for improving the efficiency of excitonic devices, where the EM mode could be provided by plasmonic structures to enable fully integrated nanometer-scale devices. We note that related results have simultaneously been obtained by Schachenmayer et al. [39].

We thank T. Ebbesen, G. Pupillo, C. Genes, and L. Martín Moreno for fruitful discussions, and E. Moreno and A. Delga for a careful reading of the manuscript. This work has been funded by the European Research Council (ERC2011-AdG Proposal No. 290981), the Spanish MINECO (Grant No. MAT2011-28581-C02-01), and by the European Union Seventh Framework Programme (Grant No. FP7-PEOPLE-2013-CIG-618229).

*johannes.feist@uam.es

fj.garcia@uam.es

[1] G. D. Scholes, G. R. Fleming, A. Olaya-Castro, and R. van Grondelle, Nat. Chem. 3, 763 (2011).

[2] G. S. Engel, T. R. Calhoun, E. L. Read, T.-K. Ahn, T. Mancal, Y.-C. Cheng, R. E. Blankenship, and G. R. Fleming, Nature (London) 446, 782 (2007).

[3] H. Lee, Y.-C. Cheng, and G. R. Fleming, Science 316, 1462 (2007).

[4] A. A. High, E. E. Novitskaya, L. V. Butov, M. Hanson, and A. C. Gossard, Science 321, 229 (2008).

[5] S. K. Saikin, A. Eisfeld, S. Valleau, and A. Aspuru-Guzik, Nanophotonics 2, 21 (2013).

[6] S. M. Menke, W. A. Luhman, and R. J. Holmes, Nat. Mater. 12, 152 (2013).

[7] Y. Dubi and M. Di Ventra, Rev. Mod. Phys. 83, 131 (2011).

[8] M. B. Plenio and S. F. Huelga, New J. Phys. 10, 113019 (2008).

[9] F. Caruso, A. W. Chin, A. Datta, S. F. Huelga, and M. B. Plenio, J. Chem. Phys. 131, 105106 (2009).

[10] J. M. Moix, M. Khasin, and J. Cao, New J. Phys. 15, 085010 (2013).

[11] S. F. Huelga and M. B. Plenio, Contemp. Phys. 54, 181 (2013).

[12] G. M. Akselrod, P. B. Deotare, N. J. Thompson, J. Lee, W. A. Tisdale, M. A. Baldo, V. M. Menon, and V. Bulović, Nat. Commun. 5, 1 (2014).

[13] J. Kasprzak, M. Richard, S. Kundermann, A. Baas, P. Jeambrun, J. M. J. Keeling, F. M. Marchetti, M. H. Szymaska, R. André, J. L. Staehli, V. Savona, P. B. Littlewood, B. Deveaud, and L.S. Dang, Nature (London) 443, 409
(2006); R. Balili, V. Hartwell, D. Snoke, L. Pfeiffer, and K. West, Science 316, 1007 (2007).

[14] S. Kéna-Cohen and S. R. Forrest, Nat. Photonics 4, 371 (2010); J. D. Plumhof, T. Stöferle, L. Mai, U. Scherf, and R. F. Mahrt, Nat. Mater. 13, 247 (2013).

[15] J. A. Hutchison, T. Schwartz, C. Genet, E. Devaux, and T. W. Ebbesen, Angew. Chemie 124, 1624 (2012).

[16] J. A. Hutchison, A. Liscio, T. Schwartz, A. CanaguierDurand, C. Genet, V. Palermo, P. Samorì, and T. W. Ebbesen, Adv. Mater. 25, 2481 (2013).

[17] D. M. Coles, N. Somaschi, P. Michetti, C. Clark, P. G. Lagoudakis, P. G. Savvidis, and D. G. Lidzey, Nat. Mater. 13, 712 (2014).

[18] D. G. Lidzey, D. D. C. Bradley, M. S. Skolnick, T. Virgili, S. Walker, and D. M. Whittaker, Nature (London) 395, 53 (1998).

[19] T. Schwartz, J. A. Hutchison, C. Genet, and T. W. Ebbesen, Phys. Rev. Lett. 106, 196405 (2011).

[20] S. Kéna-Cohen, S. A. Maier, and D. D. C. Bradley, Adv. Opt. Mater. 1, 827 (2013).

[21] J. Bellessa, C. Bonnand, J. C. Plenet, and J. Mugnier, Phys. Rev. Lett. 93, 036404 (2004).

[22] J. Dintinger, S. Klein, F. Bustos, W. L. Barnes, and T. W. Ebbesen, Phys. Rev. B 71, 035424 (2005).

[23] T. K. Hakala, J. J. Toppari, A. Kuzyk, M. Pettersson, H. Tikkanen, H. Kunttu, and P. Törmä, Phys. Rev. Lett. 103, 053602 (2009).

[24] P. Vasa, R. Pomraenke, G. Cirmi, E. De Re, W. Wang, S. Schwieger, D. Leipold, E. Runge, G. Cerullo, and C. Lienau, ACS Nano 4, 7559 (2010).

[25] S. R. K. Rodriguez, J. Feist, M. A. Verschuuren, F. J. García Vidal, and J.GómezRivas, Phys. Rev.Lett.111, 166802(2013).

[26] E. Orgiu, J. George, J. Hutchison, E. Devaux, J. F. Dayen, B. Doudin, F. Stellacci, C. Genet, P. Samorì, and T. W. Ebbesen, arXiv:1409.1900.

[27] S. Haze, Y. Tateishi, A. Noguchi, K. Toyoda, and S. Urabe, Phys. Rev. A 85, 031401(R) (2012).

[28] M. Ramm, T. Pruttivarasin, and H. Häffner, New J. Phys. 16, 063062 (2014)

[29] E. I. Rashba, Synth. Met. 64, 255 (1994).

[30] V. Agranovich and A. Kamchatnov, Chem. Phys. 245, 175 (1999).

[31] D. Manzano, M. Tiersch, A. Asadian, and H. J. Briegel, Phys. Rev. E 86, 061118 (2012).

[32] J. R. Johansson, P. D. Nation, and F. Nori, Comput. Phys. Commun. 184, 1234 (2013).

[33] J. Moll, S. Daehne, J. R. Durrant, and D. A. Wiersma, J. Chem. Phys. 102, 6362 (1995).

[34] S. Valleau, S. K. Saikin, M.-H. Yung, and A. Aspuru Guzik, J. Chem. Phys. 137, 034109 (2012).

[35] T. Schwartz, J. A. Hutchison, J. Léonard, C. Genet, S. Haacke, and T. W. Ebbesen, ChemPhysChem 14, 125 (2013).

[36] S. Wang, T. Chervy, J. George, J. A. Hutchison, C. Genet, and T. W. Ebbesen, J. Phys. Chem. Lett. 5, 1433 (2014).

[37] P. W. Anderson, D. J. Thouless, E. Abrahams, and D. S. Fisher, Phys. Rev. B 22, 3519 (1980).

[38] See Supplemental Material at http://link.aps.org/ supplemental/10.1103/PhysRevLett.114.196402 for details on the behavior under coherent driving and the analytical solution of the simplified model.

[39] J. Schachenmayer, C. Genes, E. Tignone, and G. Pupillo, following Letter, Phys. Rev. Lett. 114, 196403 (2015). 